\title{
Pilot study to determine the effectiveness of a new neck brace design for patients with amyotrophic lateral sclerosis
}

\author{
Pamela Glazener \\ Department of Rehabilitation Services, Houston Methodist Hospital, Houston, USA. \\ Correspondence: Pamela Glazener, OTR. Address: Department of Rehabilitation Services, Houston Methodist Hospital, \\ Houston, USA. Email: PGlazener@houstonmethodist.org \\ Received: March 3, 2014 \\ DOI : 10.5430/jnep.v4n6p1
}

\section{Abstract}

Background/Objective: Amyotrophic lateral sclerosis (ALS) is a progressive neurodegenerative disease. People with ALS who exhibit neck extensor weakness stop ambulating because they no longer can hold their head erect. Neck braces currently available resist or restrict lung expansion and are not an option for this population. This was a study of the impact a prototype neck brace had on quality of life, mobility, and breathing capacity of people with ALS.

Methods: Five subjects were recruited and fitted for the prototype brace. Subjects completed a demographic form, ALS-Specific Quality of Life (ALSSQOL) survey, Timed Up and Go (TUG) test, and Forced Vital Capacity (FVC) test with and without the brace. Subjects wore the brace 1 to 3 months and then repeated the measurements. Descriptive statistics were used to describe the sample and summarize the data.

Results: The initial average ALSSQOL score was 6.0. After 1 to 3 months of wearing the brace, the ALSSQOL score was 4.8. The initial FVC average showed $34.6 \%$ predicted without the brace and $37.4 \%$ predicted with the brace. After 1 to 3 months, the FVC average was $32.85 \%$ without the brace and $36 \%$ with the brace.

Conclusion: The prototype neck brace allows ALS patients with neck weakness to remain ambulatory while improving or maintaining their respiratory status. Although this pilot study had a small number of subjects, the brace was shown to be beneficial in terms of quality of life and improved respirations. Even small improvements have a large impact clinically in this patient population.

\section{Key words}

Amyotrophic lateral sclerosis, Head drop, Head drop syndrome, Neck brace, Head /neck orthosis, Neuromuscular disease

\section{Background}

Amyotrophic lateral sclerosis (ALS), also known as Lou Gehrig's disease, is a progressive neurodegenerative disease that can lead to muscular weakness. The average age of people affected by this disease ranges between 40 and 70 years old; however, it can occur at younger ages. An average of 30,000 people in the United Stated are affected by ALS at any given time and approximately 5000 new cases are diagnosed each year. ALS does not have an increased occurrence in any specific race, ethnic, or socioeconomic groups. There is no known cause or cure for ALS ${ }^{[1]}$. 
ALS causes a deterioration of upper and lower motor neurons, which can result in a variety of deficits including weakness, speech difficulty, swallowing difficulty, spasticity, weight loss, atrophy of the muscles, shortness of breath, fatigue, and eventually respiratory failure ${ }^{[2]}$. One muscle group frequently affected with weakness and fatigue are the neck extensors. Patients can lose the ability to control their head even though they may continue to be independent with ambulation (see Figure 1). However, many people with ALS who exhibit neck extensor weakness can find it difficult to ambulate due to the flexed neck position. This flexed neck position impacts the person’s ability to breathe, and causes unstable balance, fatigue, pain, and ultimately poor quality of life ${ }^{[3]}$. People with neck weakness must rely on external supports for head control, such as a neck brace or a high-backed wheelchair with head support.

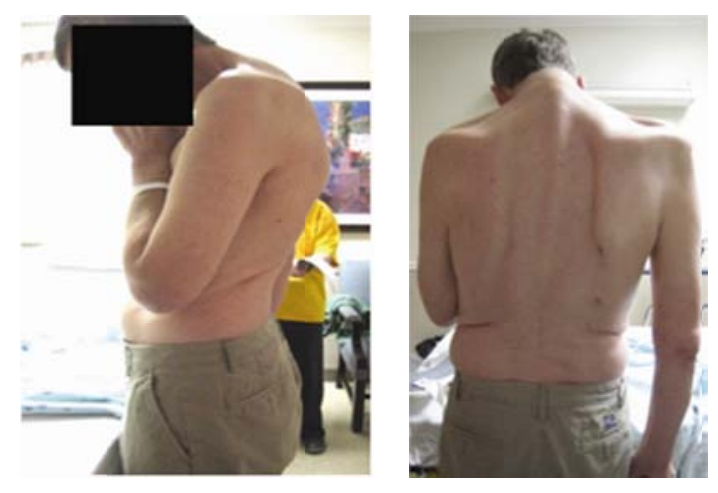

Figure 1. ALS patient with neck weakness

Multiple cervical collars are available on the market, including but not limited to the Aspen collars (Aspen Medical Products, Inc; Irvine, CA), Head Master collars (Symmetric Designs; Salt Springs Island, BC, Canada), Lerman noninvasive halo (Cascade Orthopedics; Chico, CA), Minerva brace (Cascade Orthopedics; Chico, CA), SOMI brace (Cascade Orthopedics; Chico, CA), and the MND Oxford collar (Salts \& Sons Ltd; Birmingham, England) (see Figure 2). All the current products consist of posterior support, anterior chin support, and a chest or back strap if indicated. The non-invasive halo does not consist of a chin support; however, the brace is secured with a total contact chest strap and plate. Because of the respiratory and diaphragm weakness caused by ALS, braces that resist or restrict lung expansion are not an option for this patient population. Also, patients have difficulty speaking and eating in braces with mandible support. Thus, the optimal goal for an ALS neck brace is to position the head in slight flexion without chin support and stabilize the brace posteriorly with a diaphragm-assist strap secured around the lower abdominal area (below the diaphragm). In the present study, a prototype neck brace (see Figure 3) was designed to support the head posteriorly with no restriction of the chest or mandible area. Improvements in balance, mobility, and breathing were expected with the wearing of the prototype neck brace by persons with ALS, thereby enhancing their quality of life.

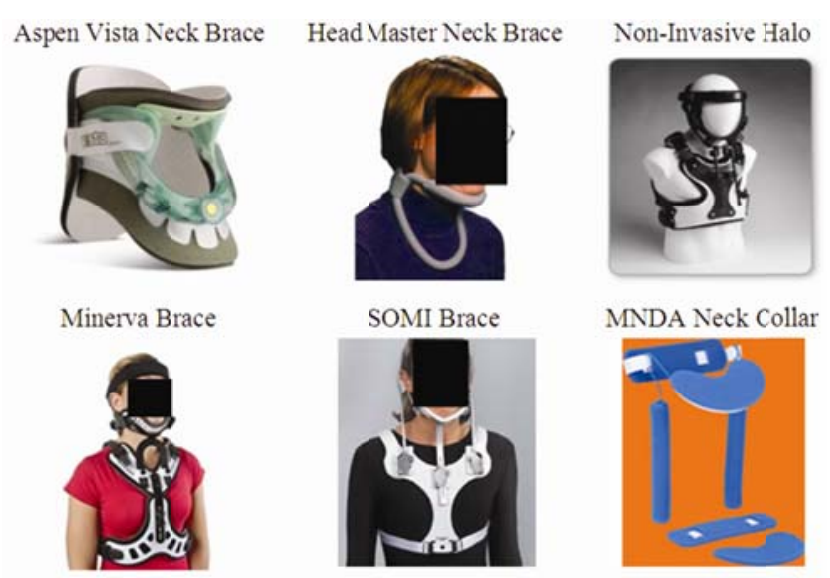

Figure 2. Examples of neck braces and collars 

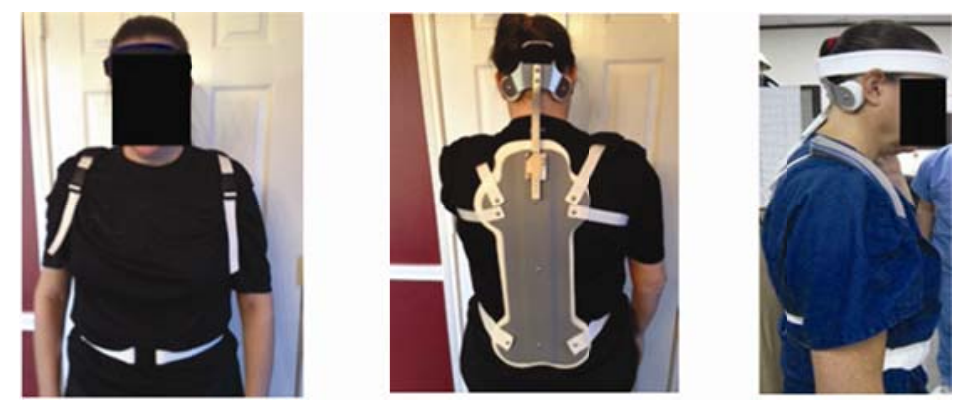

Figure 3. Prototype Neck Brace

\section{Methodology}

A pilot study was initiated to determine the effects of a prototype neck brace on the quality of life, balance, mobility, and breathing capacity of people with ALS. The study protocol was approved by the Houston Methodist Hospital IRB committee. We aimed to recruit 5 to 10 prospective subjects from the Houston Methodist Hospital Neurology ALS clinic in Houston, TX. Subjects had to have a diagnosis of ALS, have manual muscle test strength in the neck extensors of 3/5 or less, be at least 18 years of age, and be able to ambulate a minimum of 20 feet without assistance. A custom cervical thoracic lumbar orthosis was made for each subject. The orthosis consisted of a head piece connected by a joint at the C5-7 level to a dorsal lumbar-thoracic shell that was secured by a shoulder harness and abdominal strap. Once the brace was fitted to the subject, data were collected at the Houston Methodist Hospital Neurology ALS clinic.

Upon initial enrollment in the study, a demographic data form was completed for each subject that included the subject's age, gender, ethnicity, date of diagnosis or onset of disease, and neck flexor/extensor muscle strength. Before receiving and wearing the brace, the subjects were required to complete an ALS-Specific Quality of Life Questionnaire (ALSSQOL) ${ }^{[4]}$. This questionnaire is an 11-item instrument with a rating scale of 0-10 that measures a wide range of variables addressing overall quality of life, activities of daily living, pain, and breathing.

Once the subject received the brace, data were collected two times. Mobility and breathing capacity measurements were collected both with and without the subject wearing the brace. The subjects completed the Timed Up and Go (TUG) functional mobility test ${ }^{[5]}$. The TUG is a basic functional mobility measurement that is timed. The test assesses the patient's ability to stand from a chair, walk $3 \mathrm{~m}$, and return to the chair. The subject is not allowed to have any assistance with any part of the task. Results are correlated with gait speed and balance. Last, the subjects completed the forced vital capacity (FVC) lung test ${ }^{[6]}$. The FVC is a test that measures maximum breath volume and respiratory sufficiency. The FVC is determined by the power of the respiratory muscles, elastic properties of the chest wall, sex, and body size.

Once initial data were obtained, the subjects wore the brace for 1 to 3 months. The subject then returned to the Houston Methodist Hospital ALS clinic and again completed the ALSSQOL, TUG and FVC with and without the brace. The subjects served as their own controls. Control measures included the pretrial ALSSQOL, TUG, and FVC with the brace and the three measurements without the brace. These were important control measures because people with this medical condition deteriorate over time.

\section{Results and discussion}

A total of seven subjects enrolled in this pilot study; however, data were obtained from only five subjects. Two subjects did not complete the study due to rapid progression of their disease. The five participating subjects were white males whose ages ranged from 49 to 71 years (mean: 60.4 years). Initial neck extensor measurements ranged from 0/5 to 3-/5 
strength; however, the measurements for all subjects after 1 to 3 months was 0/5 strength. The data were analyzed as a descriptive study due to the small number of subjects.

The overall ALSSQOL average score was 6.0 on a 0 to 10 scale before the subjects received the prototype brace, and the average score after the subjects had worn the brace for 1 to 3 months was 4.8. A decline in quality of life is expected over time in the ALS population (see Table 1). The TUG test data were incomplete because two subjects were unable to stand from the chair without assistance. These two subjects therefore scored zero. However, once the subjects were standing, they were able to complete the task of walking $3 \mathrm{~m}$ and back. The initial FVC averages were as follows:without the brace, $1.57 \mathrm{~L}$ (34.6\% predicted), and with the brace, $1.76 \mathrm{~L}$ (37.4\% predicted). The final FVC averages were as follows: without the brace, $1.64 \mathrm{~L}$ (32.85\% predicted), and with the brace, $1.71 \mathrm{~L}$, (36\% predicted) (see Table 2).

Table 1. Overall quality of life scores and quality of life breathing scores $(n=5)$

\begin{tabular}{llllll}
\hline \multirow{2}{*}{ Subject No. } & \multicolumn{2}{l}{ Quality of Life Overall Scores } & & \multicolumn{2}{l}{ Quality of Life Breathing Scores } \\
\cline { 2 - 3 } \cline { 5 - 6 } & Initial & Final & 1 & & Initial \\
\hline 1 & 5 & 7 & 6 & 3 & 3 \\
2 & 7 & 7 & 5 & 8 \\
3 & 6 & 3 & 8 & 1 \\
5 & 6 & 6 & 4 & 6 \\
\hline
\end{tabular}

Table 2. Forced Vital Capacity (FVC) Scores $(n=5)$

\begin{tabular}{|c|c|c|c|c|}
\hline \multirow{2}{*}{ Subject No. } & \multicolumn{2}{|c|}{ FVC - Initial (\% Predicted) } & \multicolumn{2}{|c|}{ FVC - Final (\% Predicted) } \\
\hline & Without brace & With brace & Without brace & With brace \\
\hline 1 & $25 \%$ & $29 \%$ & $41 \%$ & $44 \%$ \\
\hline 2 & $31 \%$ & $34 \%$ & $23 \%$ & $33 \%$ \\
\hline 3 & $38 \%$ & $45 \%$ & $21 \%$ & $21 \%$ \\
\hline 4 & $16 \%$ & $17 \%$ & $20 \%$ & $20 \%$ \\
\hline 5 & $63 \%$ & $62 \%$ & $59 \%$ & $62 \%$ \\
\hline
\end{tabular}

ALS is a challenging disease for patients and medical professionals. The disease has no cure and no medical technology is available to slow the disease progression. Quality of life in patients with ALS is a large focus of treatment. The data from the quality of life survey in the present study indicated that three out of five subjects did not report a change in their overall quality of life, whereas one subject reported slight improvement. When asked to rate how their breathing was in relation to their quality of life, 3 of 5 subjects again reported no change or an improvement. One subject did experience a rapid progression in respiratory muscle deterioration and required an AVAP ventilator 24 hours per day by the end of the study. These results are clinically significant, however, because the disease causes a progressive decline in function and respiratory compromise.

Although the TUG mobility data were incomplete because two subjects could not complete the test independently, the patients reported that the brace was comfortable and useful during ambulation. No falls occurred when the patients were ambulating and wearing the brace. The subjects reported that the brace improved their visual orientation when ambulating and assisted with improving their posture. The subjects also verbalized that it was easier to breathe when wearing the brace due to their improved upright posture.

The FVC data showed clinical improvement in breathing. The normal FVC predicted percentage for males is $71.5 \%$ and higher. If a person has less than $50 \%$ of predicted, that person qualifies for a BIPAP or AVAPs machine to assist with their breathing. Any breathing percentage below $20 \%$ indicates a risk of needing a ventilator. All the subjects in this pilot study 
initially and months later remained the same or showed improved breathing when wearing the brace compared with when not wearing the brace, with the exception of one subject who initially showed a slight decrease in respiratory function.

Although this pilot study included a small number of subjects, the brace was shown to be beneficial in terms of quality of life, improved posture, and improved respirations. The improvements were small, but such changes have a large impact clinically in this patient population. The purpose of this brace design is to provide full head support while improving a person's ability to walk, eat, speak and breathe by not being restricted at the mandible or chest. This brace is now being used at the Houston Methodist Hospital ALS clinic as a standard of care for head drop syndrome in patients with ALS.

\section{Conclusion}

ALS is a progressive disease with no cure. The number one cause of death in the ALS patient is respiratory compromise. The focus of many medical professionals is to improve the person's quality of life, function, and respiratory abilities. This prototype neck brace allows ALS patients to remain ambulatory while improving or maintaining their respiratory status. More research needs to be completed on the many different benefits of a neck brace of this kind.

\section{References}

[1] Muscular Dystrophy Association. Facts About Amyotrophic Lateral Sclerosis 2011.

[2] Gourie-Devi, M., Nalini, A., and Sandhy, S. Early or late appearance of “dropped head syndrome” in amyotrophic lateral sclerosis. Journal of Neurology, Neurosurgery, Psychiatry. 2003; 4: 683-686. http://dx.doi.org/10.1136/jnnp.74.5.683

[3] Pinto, S. and Carvalho, M. Motor responses of the sternocleidomastoid muscle in patients with amyotrophic lateral sclerosis. Muscle \& Nerve. 2008; 38: 1312-1317. http://dx.doi.org/10.1002/mus.21109

[4] Felgoise, S.H., Walsh, S.M., Stephens, H.E., Brothers, A., and Simmons, Z. The ALS Specific Quality of Life - Revised (ALSSQOL-R) User’s Guide. Dept of Neurology. The Penn State University College of Medicine, Hershey, PA. 2011.

[5] Podsiadlo, D., Richardson, S. The Time "Up \& Go”: A Test of Basic Functional Mobility for Frail Elderly Persons. Journal of the American Geriatrics Society. 1991; 39(2): 142-148.

[6] McCormack, M.C. and Enright, P.C. Overview of Pulmonary Function Testing in Adults. UpTo Date. 8/2013. Available from: http://www.uptodate.com/contents/overview-of-pulmonary-function-testing-in-adults? 